\title{
PUBLIC HEALTH IMPACT OF RAPID IDENTIFICATION OF EPIDEMIOLOGICALLY IMPORTANT CHARACTERISTICS OF SALMONELLA SPP. BY MALDI-TOF MS

\author{
Martin Novak $^{1}$, Tatiana Kochlanova ${ }^{2}$, Anna Tvrda ${ }^{3}$, Nina Kotlebova ${ }^{4}$
}

\begin{abstract}
:
Introduction: Infections caused by Salmonella are an ongoing worldwide public health problem, often found as a source of nosocomial infections, which cause significant socio-economic burdens. Salmonella is a major food-borne pathogen causing primarily gastrointestinal diseases as well as other localized and systemic infections and extraintestinal complications. MALDITOF MS is a new method used by clinical laboratories for rapid, reliable, cost-effective and user-friendly diagnosis of many medical important bacteria of public health interest. The use of this technique improves early identification of genus Salmonella on the species, subspecies and even serovar level, which has a positive impact on public health.

Objectives: The aim of the study was to evaluate the importance of MALDI-TOF mass spectrometry for rapid identification of epidemiologically important Salmonella serovars. Based on the latest knowledge about specific biomarker molecules the possibility to identify Salmonella enterica subsp. enterica serovar Enteritidis was verified, which is one of the most common serovars present in Europe associated with gastrointestinal diseases. For serovar Enteritidis a unique mass peak at $\mathrm{m} / \mathrm{z} 6,036$ was used

Methods: 140 clinical Salmonella isolates were collected from January to October 2017. Serotyping of Salmonella species, subspecies and serovars was performed by slide agglutination technique: 139 isolates were identified as Salmonella enterica subsp. enterica and one isolate as Salmonella enterica subsp. diarizonae (IIIb). From 139 isolates of Salmonella enterica subsp. enterica the following serovars were detected: 108 Enteritidis, 12 Typhimurium, 6 Infantis, 3 Agona, 3 Derby, 7 others. All isolates were identified also by MALDI-TOF MS as Salmonella spp. For all isolates a unique mass peak at m/z 6.036 was used, which is considered to be relevant for serovar Enteritidis according to the most recent known data.

Results: 103 isolates from a total of 108 slide agglutination positive isolates for serovar Enteritidis showed a specific mass signal at $\mathrm{m} / \mathrm{z} 6,036(+/-) .5$ isolates did not contain this specific protein. After repeated analysis from re-culture, the specific protein was found also in the remaining 5 isolates. 32 serovars other than Enteritidis did not contain this specific biomarker molecule.

Conclusion: We can confirm that MALDI-TOF MS is a rapid and reliable method to identify of the most common serovar Salmonella Enteritidis based on the diagnostic marker peak at $\mathrm{m} / \mathrm{z} 6,036$ identified by recent studies. This unique mass signal showed $100 \%$ specificity and $95 \%$ sensitivity for Enteritidis serovar in our study. We can conclude that the determination of this frequently present serovar is significantly accelerated by MALDI-TOF MS. The rapid and reliable diagnosis is important for the early treatment and prevention of the spread of salmonellosis with a positive impact on public health.
\end{abstract}

UDC Classification: 616-093, DOI: 10.12955/cbup.v7.1455

Keywords: Salmonella spp., MALDI-TOF MS, public health, infection, identification

\section{Introduction}

Infections caused by Salmonella are an ongoing worldwide public health problem, often found as a source of nosocomial infections, which cause significant socio-economic burdens (Kuhns et al., 2012; Hiller et al., 2019). In terms of distribution, Salmonella are extensively represented within the environment as major zoonotic food-borne pathogens, causing outbreaks (Dieckmann \& Malorny, 2011). As Besser (2018) writes, Salmonella epidemiology is facing important challenges and new opportunities due to the rapid adoption of culture independent diagnostic test panels by clinical laboratories.

\section{Salmonella identification}

The genus Salmonella consists of two species, Salmonella enterica and Salmonella bongori. The different $S$. enterica subspecies are indicated by symbols and six subspecies are known: $S$. enterica subsp. enterica (I), S. enterica subsp. salamae (II), S. enterica subsp. arizonae (IIIa), S. enterica subsp. diarizonae (IIIb), S. enterica subsp. houtenae (IV), and S. enterica subsp. indica (VI). This nomenclature

\footnotetext{
${ }^{1}$ Jessenius Faculty of Medicine in Martin, Comenius University in Bratislava, Martin, Slovakia, martin4novak@gmail.com

2 Jessenius Faculty of Medicine in Martin, Comenius University in Bratislava, Martin, Slovakia, tatianakochlanova@gmail.com

${ }^{3}$ Clinical Biochemistry, JSC, Department of Clinical Microbiology, Zilina, Slovakia, atvrda@gmail.com

${ }^{4}$ Jessenius Faculty of Medicine in Martin, Comenius University in Bratislava, Martin, Slovakia, nina.kotlebova@gmail.com
} 
reflects the present understanding of Salmonella taxonomy (Issenhuth-Jeanjean et al., 2014; Ryan et al., 2017).

\section{Serotyping of Salmonella}

Currently, 2,610 different Salmonella serovars have been recognized according to the WhiteKauffmann-Le Minor classification scheme (Dieckmann \& Malorny, 2011). As Guibourdenche et al. (2010) write, this serotyping scheme is a gold standard for the identification of Salmonella below the subspecies level. This method is based on a serotyping of the somatic $\mathrm{O}$ antigens (lipopolysaccharid), flagellar $\mathrm{H} 1$ and $\mathrm{H} 2$ antigens (flagellar proteins) and capsular Vi antigens (capsular polysaccharid) by slide agglutination (Grimont \& Weill, 2007; Guibourdenche et al., 2010; Issenhuth-Jeanjean et al., 2014; Ryan et al., 2017). Traditional serology and the White-Kauffmann scheme have been accepted worldwide for Salmonella serotyping (Ibrahim \& Morin, 2018).

\section{Identification of Salmonella by MALDI-TOF MS}

Matrix-assisted laser desorption ionization-time of flight mass spectrometry (MALDI-TOF MS) is a new method used for rapid and reliable diagnosis of many medical important bacteria of public health interest. As Kostrzewa (2018) writes, the MALDI Biotyper has significantly improved clinical microbiology in the area of microorganism identification. The introduction of the MALDI Biotyper in laboratories substantially changed microbiology practice. This has been called revolutionary, because rapid microbiological diagnostics have been shown to have a positive effect on patient management (Kostrzewa, 2018, Idelevich et al., 2018). A mass spectrometer is composed of an ion source to ionize and transfer sample molecules ions into a gas phase, a mass analyzer that separate ions according to their mass-to-charge ratio $(\mathrm{m} / \mathrm{z})$ and a detection device to monitor the separated ions. Samples are prepared by mixing with a matrix which results in the crystallization of the sample within the matrix (Croxatto et al., 2012). MALDI-TOF MS analysis identifies bacteria on the basis of their protein profile. The identification is based on a comparison of the mass spectra of bacterial proteins with the known protein reference spectra in the database (Croxatto et al., 2012; Neuschlova et al., 2017).

\section{Modern methods in clinical microbiology}

Nowadays, several modern methods are used in clinical microbiological laboratories, that present a costeffective, rapid and reliable way of identifying of a broad spectrum of pathogens causing many local and systematic infections in gastrointestinal, urogenital, respiratory tract and other areas of the body (Kompanikova et al., 2017). New methods based on microarrays as well as on the detection of protein spectra by MALDI-TOF mass spectrophotometry (MS) are suitable for routine clinical use (Neuschlova et al, 2017; Kompanikova et al., 2017). Automated analysis of protein spectra from different microbial populations is an important tool for epidemiological studies and may have an impact on public health (Rodríguez-Sánchez et al., 2019). Rapid and reliable identification of pathogens, including Salmonella sp., is important for surveillance, prevention, and control of food-borne diseases (Dieckmann et al., 2008).

In routine diagnosis by MALDI-TOF MS Salmonella can be identified on a genus level only. Lower level identification is not possible with conventional identification because of the close proximity of protein spectra (Bilecent et al., 2015). The prevailing approach for bacterial identification in MALDITOF MS is the fingerprinting method, which compares the mass spectra of target isolates with those of well characterized reference strains in commercially available protein spectra databases (Fothergill et al., 2013; Singhal et al., 2015). Using whole cells, up to 30 constant peaks whose molecular weight is in the range of 4,000-13,000 Da are detected in mass spectra. Salmonella is one of the epidemiologically important and clinically relevant pathogens for which the number of these peaks is sufficient to identify at the genus and species level only (Dieckmann et al., 2008; Dieckmann \& Malorna, 2011). Using a decision tree based on the presence/absence of specific peaks, corresponding mainly to ribosomal proteins, the authors achieved correct identification of the most commonly encountered S. enterica subsp. enterica serovars with $100 \%$ sensitivity and specificity. More recently, a study using similar peaks allowed correct identification of $94 \%$ S. enterica subsp. enterica serovars assignment using a set of 12 species-specific peaks (Ojima-Kato et al., 2017; Rodríguez-Sánchez et al., 2019).

\section{Objectives}

The aim of the study was to evaluate the importance of MALDI-TOF MS for rapid identification of epidemiologically important Salmonella serovars, based on serovar specific biomarker molecules and 
point out of this rapid identification with public health importance. Salmonella enterica subsp. enterica serovar Enteritidis is the most common serovar in Europe associated with gastrointestinal diseases. Our study was based on the study by Dieckmann and Malorna (2011), who selected serovar specific biomarker molecules for the rapid identification and classification of the most frequently occurring Salmonella enterica serovars. For serovar Enteritidis a unique mass peak at mass-to-charge ratio $\mathrm{m} / \mathrm{z}$ 6,036 was used, which responds to a not yet characterized protein. The presence of the same protein at $\mathrm{m} / \mathrm{z}$ 6,009 indicates another serovar (Dieckmann et al., 2008; Dieckmann \& Malorny, 2011).

\section{Material and methods}

A total of 140 clinical Salmonella isolates were collected from January to October 2017, on Clinical Biochemistry, JSC, in Slovakia. All of these isolates were identified by MALDI-TOF MS as Salmonella spp. Serotyping of Salmonella species, subspecies and serovars was performed by the slide agglutination technique. 139 isolates were identified as Salmonella enterica subsp. enterica (I) and one isolate as Salmonella enterica subsp. diarizonae (IIIb). From 139 isolates of Salmonella enterica subsp. enterica the following serovars were detected: 108 Enteritidis, 12 Typhimurium, 6 Infantis, 3 Agona, 3 Derby, and 7 others. All isolates were identified also by MALDI-TOF MS as Salmonella spp. For all isolates a unique mass peak at $\mathrm{m} / \mathrm{z}$ 6,036 was used, which is considered to be relevant for serovar Enteritidis according to the most recent known data. All Salmonella came from clinical material. Patient's rectal swabs were inoculated directly on MacConkey agar and selenite cystine broth. The strains were grown for 24 hours at $37^{\circ} \mathrm{C}$. The swab was then propagated in $24 \mathrm{~h}$ selenite at $37^{\circ} \mathrm{C}$. After 24 hours of cultivation in the broth, the swab was inoculated on deoxycholate agar, where the strains were grown at $37^{\circ} \mathrm{C}$ for 24 hours. Salmonella identification was performed routinely, and whole cell identification was performed directly from the plate by MALDI-TOF MS without extraction. The samples were mixed with matrix $\alpha$-cyano-4-hydroxycinnamic acid, as recommended by the manufacturer Bruker Daltonics, Bremen, Germany. Samples with a matrix resulted in the crystallization of the sample within the matrix. MALDI-TOF MS identification of isolates was performed on a Microflex LT linear spectrometer with a Bruker microSCOUT ion source and a TOF flight time analyzer. The setup proposed by the manufacturer for routine identification was used. A nitrogen laser $(\lambda=337 \mathrm{~nm})$, a positive linear mode $(20 \mathrm{kV}, \mathrm{m} / \mathrm{z} 2000-20,000)$ under the control of FlexControl software 3.3 was used to obtain ions at 2-20 $\mathrm{kDa}$. Each spectrum was obtained by averaging up to 240 laser shots. Automated analysis of the protein spectrum was performed by MALDI BioTyper version 3.0, comparing the unknown spectrum with the database. In order to find a unique peak, it was necessary to regain the protein profile, which was then analyzed in the Flexanalysis (Bruker) program to determine the presence of the peak. After identifying isolates as Salmonella spp. the isolates were inoculated on TSI agar and cultivated for 24 hours $37^{\circ} \mathrm{C}$. The next day they were agglutinated according to the White-Kauffmann-Le Minor scheme, using agglutination sera (Oxoid).

\section{Results}

A total of 140 clinical Salmonella isolates were identified by MALDI-TOF MS as Salmonella spp. Serotyping of Salmonella was performed by slide agglutination technique. 139 isolates were identified (Table 1) as Salmonella enterica subsp. enterica (I) and one isolate as Salmonella enterica subsp. diarizonae (IIIb). From 139 isolates of Salmonella enterica subsp. enterica the following serovars were detected: 108 Enteritidis, 12 Typhimurium, 6 Infantis, 3 Agona, 3 Derby, and 7 others (Table 2, Figure 1). All isolates were identified also by MALDI-TOF MS as Salmonella spp. For all isolates a unique mass peak at m/z 6,036 was used, which is considered to be relevant for serovar Enteritidis according to the most recent known data.

Data of mass spectra were exported into Microsoft Excel and were sorted by weight of peaks (m/z). 103 isolates from a total of 108 slide agglutination positive isolates for serovar Enteritidis showed a specific mass signal at $\mathrm{m} / \mathrm{z}$ 6,036 (+6 Da/-8 Da). 5 isolates did not contain this specific protein. After repeated analysis from re-culture, the specific protein was found also in the remaining 5 isolates. 32 serovars other than Enteritidis did not contain this specific biomarker molecule. Mass peaks (m/z) by whole cell MALDI-TOF MS of Salmonella enterica subsp. enterica detected by serovar Enteritidis varied in our study from 6,026 to 6,040 (Table 3, Figure 2). Median of the mass values was at m/z 6,034. Mass values for peaks observed in spectra of tested strains were within a mass tolerance window $(+6 \mathrm{Da} /-8 \mathrm{Da})$. A precision of the mass/charge $(\mathrm{m} / \mathrm{z})$ value was $0.13 \%$ in the downward direction and $0.10 \%$ in the upward 
direction. $100 \%$ specificity and $95 \%$ sensitivity showed this unique mass signal for Enteritidis serovar in our study.

\begin{tabular}{|l|l|c|}
\hline Table 1: Present number of serovars in species and subspecies of Salmonella \\
\hline \multirow{4}{*}{ Salmonella enterica } & number of isolates \\
\cline { 2 - 3 } & subsp. enterica (I) & 139 \\
\cline { 2 - 3 } & subsp. diarizonae (IIIb) & 1 \\
\cline { 2 - 3 } & Total number & $\mathbf{1 4 0}$ \\
\hline Source: Authors & \\
\hline
\end{tabular}

Table 2: Present number of serovars in species and subspecies of Salmonella enterica subsp. enterica

\begin{tabular}{|l|l|c|}
\hline & Salmonella enterica subsp. enterica & number of isolates \\
\cline { 2 - 3 } & serovar Enteritidis & 108 \\
\cline { 2 - 3 } & serovar Typhimurium & 12 \\
\cline { 2 - 3 } & serovar Infantis & 6 \\
\cline { 2 - 3 } & serovar Agona & 3 \\
\cline { 2 - 3 } & serovar Derby & 3 \\
\cline { 2 - 3 } & others & 7 \\
\hline & Total number & $\mathbf{1 3 9}$ \\
\hline Source: Authors
\end{tabular}

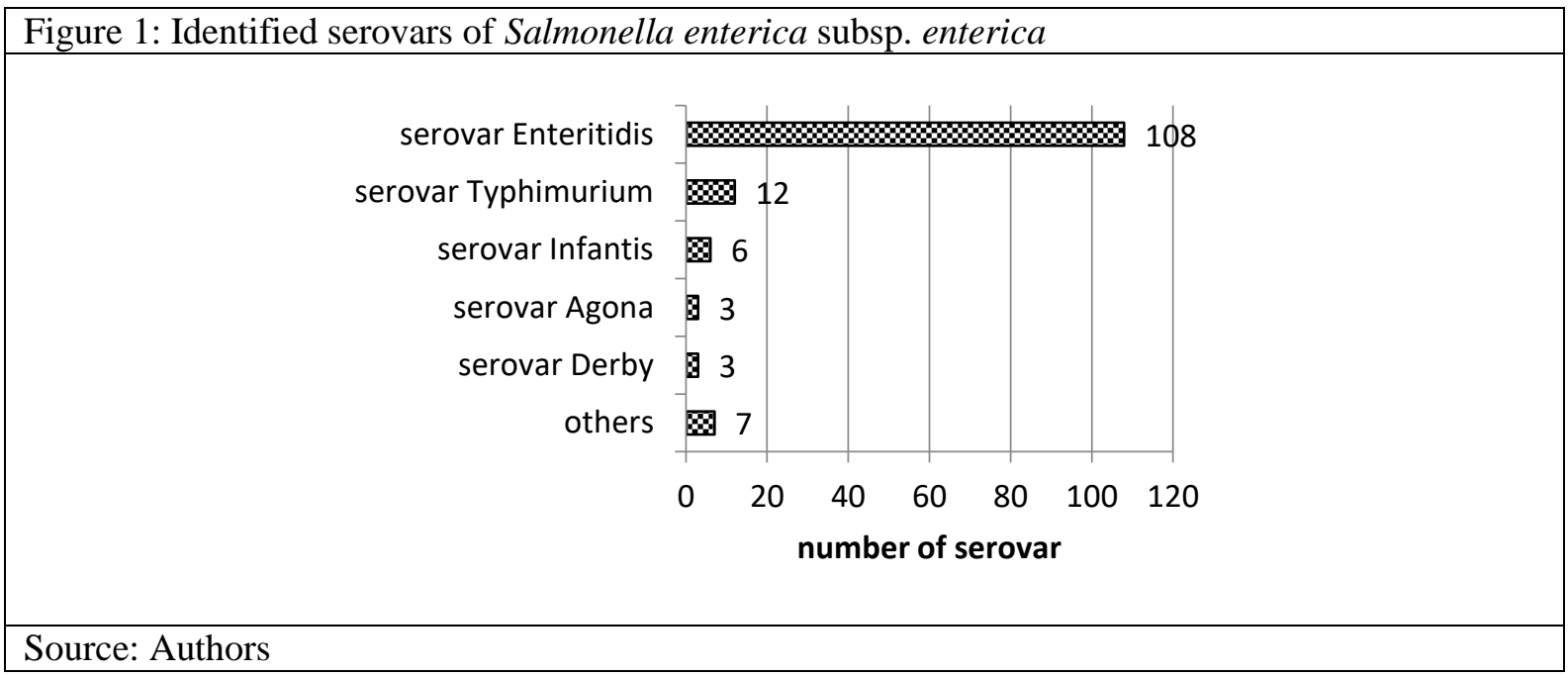

Table 3: Mass peaks (m/z) by whole cell MALDI-TOF MS of Salmonella enterica subsp. enterica detected by serovar Enteritidis

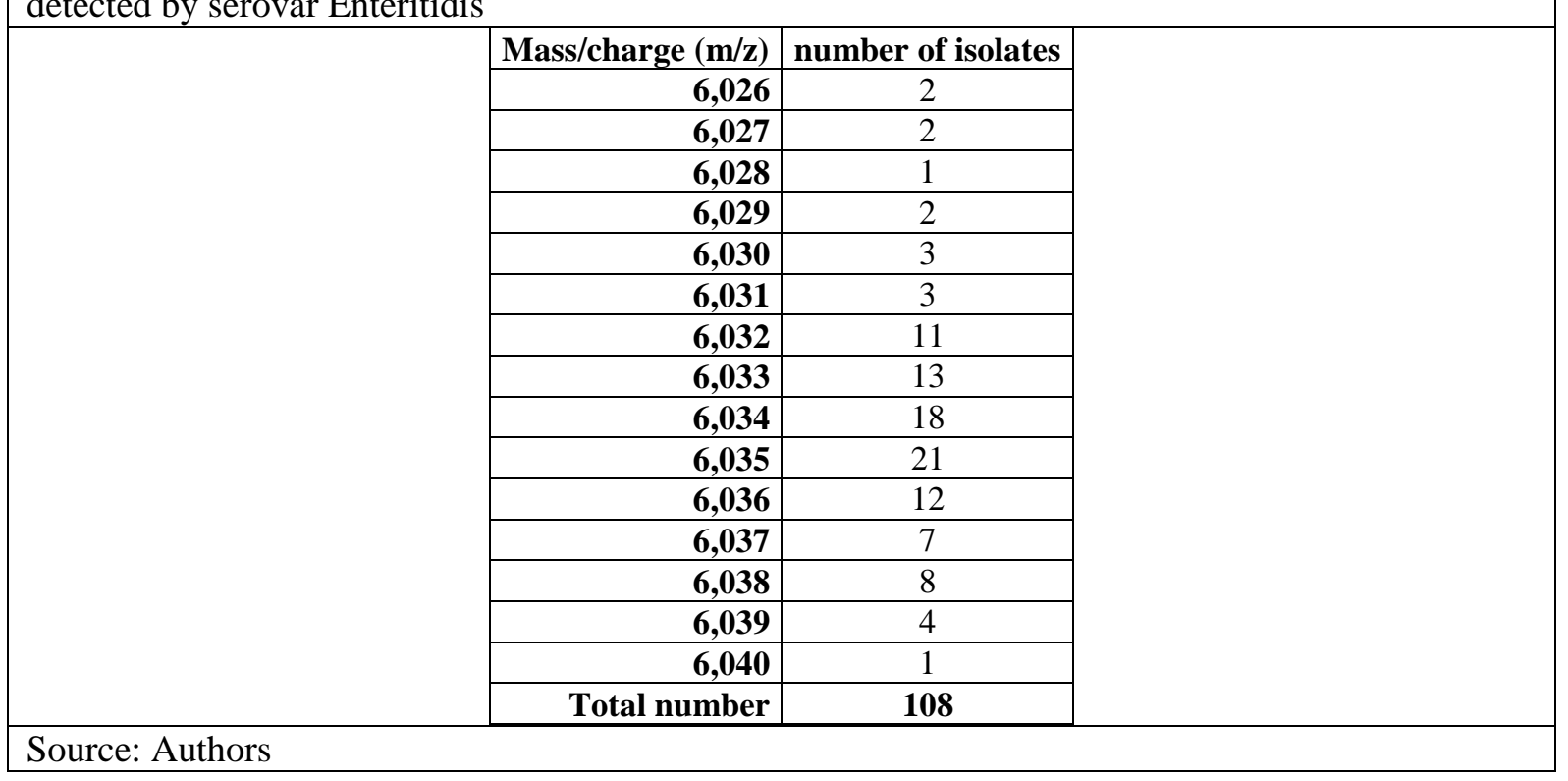




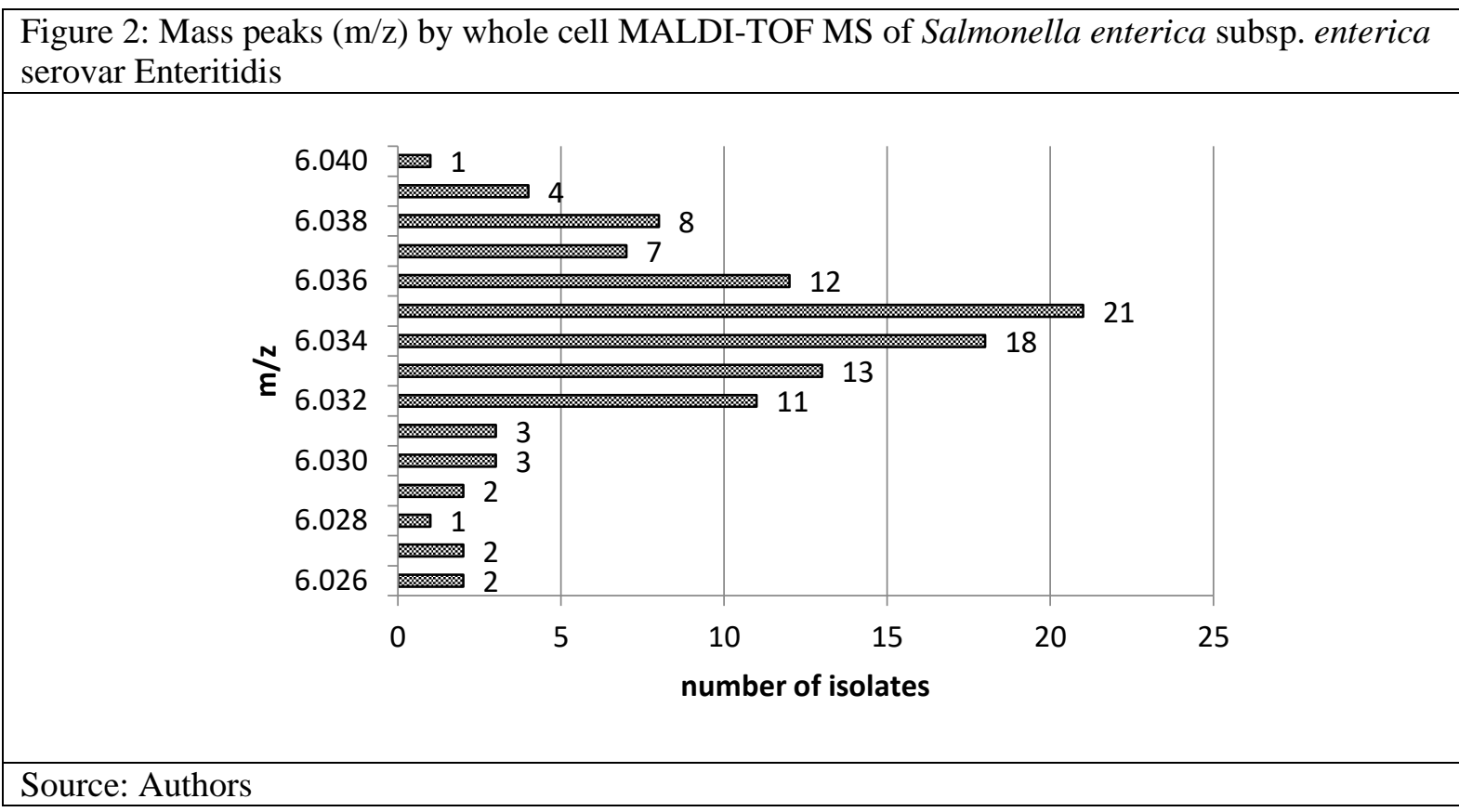

\section{Discussion}

Salmonella is a major food-borne pathogen causing primarily gastrointestinal diseases as well as other localized and systemic infections and extraintestinal complications (Kompanikova et al., 2016). MALDI-TOF MS is a revolutionary method used by clinical laboratories for rapid, reliable, costeffective and user-friendly diagnosis of many medical important bacteria of public health interest. The use of this technique improves early identification of genus Salmonella on the species, subspecies and even serovar level, which has a positive impact on public health.

Several studies confirm, that MALDI-TOF MS is a method appropriate for monitoring the epidemiological situation with regard to virulent and highly resistant pathogens among which Salmonella belongs (Rodríguez-Sánchez et al., 2019). Identification and diagnosis of affected patients as well as implementation of isolation measures would be greatly facilitated in the case of routine introduction of rapid protein acquisition and analysis, which has a positive impact on patient management and public health (Idelevich et al., 2018; Rodríguez-Sánchez et al., 2019). Preventing the spreading of infection and increasing the curative ratio is very important for the rapid detection, timely treatment and modern disease management of Salmonellosis (Tae-Hoon Kim et al., 2017; Schubert \& Kostrzewa, 2017).

According to Dieckmann and Malorny (2011), MALDI-TOF MS is the recommended method for clinical practice, based on its reliability, speed and affordability. In their study they analyzed 913 Salmonella isolates, 89 different serovars, 53 of which were identified as Salmonella Enteritidis. The unique protein was found in the $\mathrm{m} / \mathrm{z}$ region 6,036 and presented its $100 \%$ exclusivity. Sensitivity was $86.5 \%$ for this serovar. Larger mass tolerance window $( \pm 5 \mathrm{Da})$ were preliminarily assigned larger and low-intensity protein peaks (Dieckmann et al., 2008).

Kang (2017) found in his study that MALDI-TOF provides high accuracy in species-level identification, but their results show that it was limited to the type or subtype of Salmonella serovars.

As Leuschner et al. (2003) write, they analyzed $5 S$. Enteritidis isolates and found a specific peak at $\mathrm{m} / \mathrm{z}$ 6,030 . The authors analyzed the peaks manually, with a tolerance of $0.2 \%$ in both directions. This peak is probably identical to the peak $\mathrm{m} / \mathrm{z}$ 6,036 of Dieckmann and Malorny (2011). In our study a tolerance of the $\mathrm{m} / \mathrm{z} 0.13 \%$ was detected in the downward direction and $0.10 \%$ in the upward direction.

In our study we found a unique peak in 108 clinical isolates of Salmonella Enteritidis and confirmed its $100 \%$ specificity. Repeated culture and identification were performed in 5 isolates of 108 . The peak was found in all 5 cases after re-measurement. The sensitivity of the identification method was $95 \%$ in our study. Dieckmann and Malorny (2011) report sensitivity of $86.5 \%$. This number also confirms their findings that from fresh clinical material, the sensitivity of the biomarker is higher. 
Many researchers confirm that MALDI-TOF MS makes an excellent tool for monitoring the epidemiology of highly resistant or virulent pathogens as well as for outbreak detection and for screening of isolates within an outbreak or for the detection of slow-growing bacteria (Neuschlova et al., 2017; Akyar et al, 2018; Cenci et al., 2018; Rodríguez-Sánchez et al., 2019).

One of the major advantages of MALDI-TOF MS technology for identification of pathogens is the timeto-result, which is reduced from 24-48 h to less than an hour (Croxato et al., 2012).

\section{Conclusions}

We can confirm that MALDI-TOF MS is a rapid and reliable method to identify the most common serovar Salmonella Enteritidis based on the diagnostic marker peak at $\mathrm{m} / \mathrm{z}$ 6,036 identified by recent studies. This unique mass signal showed 100\% specificity and $95 \%$ sensitivity for Enteritidis serovar in our study. We can conclude that the determination of this frequently present serovar is significantly accelerated by MALDI-TOF MS. The rapid and reliable diagnosis is important for the early treatment and prevention of the spread of salmonellosis with a positive impact on public health.

\section{References}

Akyar, I., Cavzsoglu, C., Avas, M., Sürücüoglu, S., Ilki, A., Kaya, DE., Besli, Y. (2018). Evaluation of the performance of MALDI-TOF MS and DNA sequence analysis in the identification of mycobacteria species. Turk J Med Sci, 48 (6), 1351 1357. doi: https://dx.doi.org/10.3906/sag-1801-198.

Besser, J. (2018). Salmonella epidemiology: a whirlwind of change. Food Microbiol, 7, 55-59. doi: https://dx.doi.org/ 10.1016/j.fm.2017.08.018.

Bilecen, K., Yaman, G., Ciftci, U., Laleil, Y.R. (2015) Performances and Reliability of Bruker Microflex LT and VITEK MS MALDI-TOF Mass Spectrometry Systems for the Identification of Clinical Microorganisms. Biomed Res Int, $2015,516410$. doi: $10.1155 / 2015 / 516410$

Cenci, E., Luciano, E., Bucaioni, M., Rubeca, M., Cesarini, A., Bozza, S., De Socio, G.V., Mencacci, A. (2018). Evaluation of IVD 3.0 Vitek MS matrix-assisted laser desorption ionization-time of flight mass spectrometry for identification of Mycobacterium tuberculosis and nontuberculous mycobacteria and its use in routine diagnostics. Eur J Clin Microbiol Infect Dis, 37 (10), 2027-2029. doi: https://dx.doi.org/10.1007/s10096-018-3326-3.

Croxatto, A., Prodhom, G., Greub, G. (2012). Applications of MALDI-TOF mass spectrometry in clinical diagnostic microbiology. FEMS Microbiol Rev, 36, 380-407. doi: https://dx.doi.org/10.1111/j.1574-6976.2011.00298.x.

Dieckmann, R., Helmuth, R., Erhard, M., Malorny, B. (2008). Rapid Classification and Identification of Salmonellae at the Species and Subspecies Levels by Whole-Cell Matrix-Assisted Laser Desorption Ionization-Time of Flight Mass Spectrometry . Appl Environ Microbiol, 74(24), 7767-78. doi: https://dx.doi.org/10.1128/AEM.01402-08.

Dieckmann, R., Malorny, B. (2011). Rapid Screening of Epidemiologically Important Salmonella enterica subsp. enterica Serovars by Whole-Cell Matrix-Assisted Laser Desorption Ionization-Time of Flight Mass Spectrometry. Appl Envirom Microbiol, 77(12), 4136-4146. doi: https://dx.doi.org/10.1128/AEM.02418-10.

Fothergill, A., Kasinathan, V., Hyman, J., Walsh, J., Drake, T., Wang, Y. (2013). Rapid identification of bacteria and yeasts from positive-bloodculture bottles by using a lysis-filtration method and matrixassisted laser desorption ionization-time of flight mass spectrum analysis with the SARAMIS database. J Clin Microbiol, 51, 805- 809. doi: https://dx.doi.org/10.1128/JCM.02326-12.

Grimont, P.A.D., Weill, F.X. Antigenic formulae of the Salmonella serovars. ( $9^{\text {th }}$ edition)., Paris, France: WHO Collaborating Centre for Reference and Research on Salmonella, Institut Pasteur, (p. 166).

Guibourdenche, M., Roggentin, P., Mikoleit, M., Fields, P.I., Bockemuhl, J., Grimont, P.A., et al. (2010). Supplement $2003-$ 2007 (No. 47) to the White-Kauffmann-Le Minor scheme. Res Microbiol, 161, 26-29. doi: https://dx.doi.org/10.1016/j.resmic.2009.10.002.

Hiller, C.C., Lucca, V., Carvalho, D., Borsoi, A., Borges, K.A., Furian, T.Q., et al. (2019). Influence of catecholamines on biofilm formation by Salmonella Enteritidis. Microb Pathog, 130, 54-58. doi: https://dx.doi.org/10.1016/j.micpath.2019.02.032.

Ibrahim, G.M., Morin, P.M. (2018). Salmonella Serotyping Using Whole Genome Sequencing. Front Microbiol, 9, 2993. doi: https://dx.doi.org/ 10.3389/fmicb.2018.02993.

Idelevich, EA., Storck, LM., Sparbier, K., Drews, O., Kostrzewa, M., Becker, K. (2018). Rapid direct susceptibility testing from positive blood cultures by the matrix-assisted laser desorption ionization-time of flight mass spectrometry based directon-target microdroplet growth assay. J Clin Microbiol, 56(10), e00913-18. doi: https://dx.doi.org/10.1128/JCM.00913-18. Issenhuth-Jeanjean, S., Roggentin, P., Mikoleit, M., Guibourdenche, M., De Pinna, E., Nair, S., et al. (2014). Supplement 2008-2010 (no. 48) to the White-Kauffmann-Le Minor scheme. Res Microbiol, 165 (7), 526-30. doi: https://dx.doi.org/10.1016/j.resmic.2014.07.004

Kang, L., Li, N., Li, P., Zhou, Y., Gao, S., Gao, H., Xin, W., Wang, J. (2017). MALDI-TOF mass spectrometry provides high accuranxy in identification of Salmonella at species level but is limited to type or subtype Salmonella serovars. Eur J Mass Spectrom (Chichester), 23(2), 70-82. doi: https://dx.doi.org/10.1177/1469066717699216. 
Kim, T.H., Hwang, H.J., Kim, J.H. (2017). Development of a Novel, Rapid Multiplex Polymerase Chain Reaction Assay for the Detection and Differentiation of Salmonella enterica Serovars Enteritidis and Typhimurium Using Ultra-Fast Convection Polymerase Chain Reaction. Foodborne Pathog Dis, 14(10), 580-586. doi: https://dx.doi.org/10.1089/fpd.2017.2290.

Kompanikova, J., Neuschlova, M., Sadlonova, V. (2016). Special bacteriology basic laboratory tests. Martin, Slovakia: Jesseniova lekárska fakulta UK, (109 p.) ISBN 978-80-8187-019-4.

Kompanikova, J., Zumdick, A., Neuschlova, M., Sadlonova, V., Novakova, E. (2017). Microbiologic Methods in the Diagnostics of Upper Respiratory Tract Pathogens. Adv Exp Med Biol, 32, 25-31. doi:

https://dx.doi.org/10.1007/5584_2017_10.

Kostrzewa, M. (2018). Application of the MALDI Biotyper to clinical microbiology: progress and potential. Expert Rev Proteomics, 15, 193-202. doi: https://dx.doi.org/10.1080/14789450.2018.1438193.

Kuhns, M., Zautner, A.E., Rabsch, W., Zimmermann, O., Weig, M., Bader, O., Groß, U. (2012). Rapid Discrimination of Salmonella enterica Serovar Typhi from Other Serovars by MALDI-TOF Mass Spectrometry. PloS One, 7(6), e40004. doi: https://dx.doi.org/10.1371/journal.pone.0040004.

Leuschner, R.G.K., Beresford-Jones, N., Robinson, C. (2003). Difference and consensus of whole cell Salmonella enterica subsp. Enterica serovars matrix-assisted laser desorption/ionization time-of-flight mass spectrometry spectra. Lett Appl Microbiol, 38, 24-31.

Neuschlova, M., Vladarova, M., Kompanikova, J., Sadlonova, V., Novakova, E. (2017). Identification of Mycobacterium Species by MALDI-TOF Mass Spectrometry. Adv Exp Med Biol, 1021, 37-42. doi: https://dx.doi.org/10.1007/5584_2017_26.

Ojima-Kato, T., Yamamoto, N., Nagai, S., Shima, K., Akiyama, Y., Ota, J., Tamura, H. (2017). Application of proteotyping Strain Solution ${ }^{\mathrm{TM}}$ ver. 2 software and theoretically calculated mass database in MALDI-TOF MS typing of Salmonella serotype. Appl Microbiol Biotechnol, 101(23-24):8557-8569. doi: https://dx.doi.org/10.1007/s00253-017-8563-3

Rodríguez-Sánchez, B., Cercenado, E., Coste, A.T., Greub, G. (2019). Review of the impact of MALDI-TOF MS in public health and hospital hygiene. Eurosurveill, 24(4), 1800193. doi: https://dx.doi.org/10.2807/1560-7917.

Ryan, M.P., O’Dwyer, J., Adley, C.C. (2017). Evaluation of the Complex Nomenclature of the Clinically and Veterinary Significant Pathogen Salmonella. BioMed ResInt, 2017, 3782182. doi: https://dx.doi.org/10.1155/2017/3782182.

Schubert, S., Kostrzewa, M. (2017). MALDI-TOF MS in the Microbiology Laboratory: Current Trends. Curr Issues Mol Biol, 23, 17-20. doi: https://dx.doi.org/10.21775/cimb.023.017.

Singhal, N., Kumar, M., Kanaujia, P.K., Virdi, J.S. (2015). MALDI-TOF mass spectrometry: an emerging technology for microbial identification and diagnosis. Front Microbiol, 6, 791. doi: https://dx.doi.org/10.3389/fmicb.2015.00791. 